\title{
Green biosynthesis of gold nanometre scale plates using the leaf extracts from an indigenous Australian plant Eucalyptus
} macrocarpa

\author{
Gérrard Eddy Jai Poinern • Peter Chapman • Xuan Le • \\ Derek Fawcett \\ Published online: 2 July 2013 \\ (C) The Author(s) 2013. This article is published with open access at SpringerLink.com
}

\begin{abstract}
In this preliminary study, we demonstrate an environmentally friendly process for the green synthesis of gold nanometre scale particles using the leaf extract from an indigenous Australian plant Eucalyptus macrocarpa as both the stabilising agent and the reducing agent. The synthesis process is straightforward, clean and non-toxic. It also has the advantages of being performed at room temperature and does not need complex processing equipment. Formation of the gold nanometre sized particles was confirmed and characterised by UV-visible spectroscopy, X-ray diffraction, transmission electron microscopy and field emission scanning electron microscopy. The antibacterial activity of the synthesised gold particles was also quantified using the sensitivity method of Kirby-Bauer.
\end{abstract}

Keywords Gold · Nano-particles · Green synthesis ·

Antibacterial $\cdot$ Eucalyptus macrocarpa

\section{Introduction}

Metallic nanometre scale particles, in particular colloidal gold nanoparticles ( $\mathrm{Au} \mathrm{NPs}$ ), have attracted considerable interest in many fields such as medicine, biotechnology, materials science, photonics and electronics [1-4]. The size, shape and surface morphology can have a profound influence on the chemical, physical, optical and electronic properties of nano-

G. E. J. Poinern $(\bowtie) \cdot$ X. Le $\cdot$ D. Fawcett

Murdoch Applied Nanotechnology Research Group,

Department of Physics, Energy Studies, and Nanotechnology,

School of Engineering and Energy, Murdoch University,

Murdoch, WA 6150, Australia

e-mail: g.poinern@murdoch.edu.au

P. Chapman

Department of Chemistry, Curtin University of Technology,

Bentley, WA 6102, Australia materials $[5,6]$. This is indeed the case for a noble metal such as $\mathrm{Au}$, where the metal exhibits a strong surface plasmon resonance when exposed to electromagnetic radiation [7]. Until recently, producing nanoparticles involved expensive chemical and physical processes that often used toxic materials with potential hazards such as environmental toxicity, cytotoxicity and carcinogenicity [8]. An attractive alternative to the traditional manufacturing techniques used for the production of nanoparticles involves using a green environmentally friendly technology based on biological systems such as plants $[9,10]$, bacteria $[11,12]$, fungus $[13,14]$ and similar organisms $[15,16]$. Synthesising nanoparticles via biological systems offers a clean, nontoxic and environmentally friendly method with the potential to deliver a wide variety of nanoparticle types, sizes, shapes and morphologies. Out of the several biological systems mentioned above, synthesising nanoparticles via leaf extracts from plants is a relatively straight forward technique. The technique does not need any special culture preparation or isolation techniques that are normally required for bacteria and fungi based synthesis techniques.

$\mathrm{Au}$ NPs have attracted significant interest over the last decade as a potential platform for a number of biomedical applications such as biosensors [17], clinical chemistry [18], fluorescent labelling for immunoassays [19], tumour destruction via heating (hyperthermia) [20], targeted delivery of therapeutic drugs and genetic substances [21] and as antibacterial drugs [22,23]. The cells of most living organisms have dimensions in the range of 10 to $50 \mu \mathrm{m}$, while the cells' internal organelles and other sub-cellular structures are all in the sub-micron size range. The difference in size between a typical cell and a nanometre scale particle can range from a hundred times up to ten thousand times. This significant difference in size range between the cell and a nanometre scale particle gives the NP the potential to biophysically interact with biological molecules both inside and on the surface of cell [3]. Recent studies have shown that membrane 
damage and toxicity can result from the biosorption and cellular uptake of NPs by bacteria $[24,25]$. However, the processes behind NP inhibition of bacterial growth are not fully understood, but some studies have strongly suggested that the size, shape and surface modifications could influence the antibacterial properties of the NPs [25, 26].

Chrysotherapy is a branch of medicine that uses $\mathrm{Au}$ as a medicinal compound in the treatment of certain diseases such as rheumatoid arthritis. In addition, Au NPs have also been successfully used as a carrier platform for the delivery of double-stranded DNA in gene gun technology [27]. Another feature of Au NPs is their ability to passively accumulate in tumours, where their good optical and chemical properties can be used in thermal treatment therapies [28, 29]. Moreover, recent studies have shown that cancer drugs bonded to the surface of Au NPs can be more effectively targeted to tumours, thus improving delivery and minimising treatment durations and side effects of anticancer drugs [3, 30-33]. Au NPs have also attracted significant scientific interest as a new class of biomedical materials, since they can act as both antibacterial and antifungal agents capable of interacting with microorganisms to produce cellular damage [33]. The ability to induce cellular damage and the ultimate death of various strains of bacteria and yeast microorganisms has many significant health benefits. For example, both bacterial and fungal species have the ability to develop immunity against commonly used antibiotic with time. Therefore, new and more effective antimicrobial agents are needed to fight antibiotic resistant strains of microorganisms. Au NPs have the potential to be an effective antimicrobial agent, especially if they can be synthesised via a green chemistry route that ensures a clean, nontoxic and environmentally friendly method for producing NPs.

The southwest corner of the Australian continent is a global biodiversity hot spot and is also the home of the exquisite Eucalyptus macrocarpa [34]. The plant is also known as the Rose of the West or the Mottlecah and is easily recognised by its beautiful silvery foliage and red flowers as presented in Fig. 1a. And because of its attractive floral arrangement, the plant has been successfully propagated throughout the coastal region of Western Australian. The silvery grey appearance of the plants leaves are the result of nanometre scale features formed on the surface of the leaf by epicuticular waxes. These waxes give the leaves their remarkable wetting and selfcleaning properties, which enhances the plants survival in its arid climate [35].

In this paper, we report for the first time the green synthesis of stable Au NPs by the direct reduction of $\mathrm{AuCl}_{4}{ }^{-}$ions via Mottlecah leaf extracts without using conventional stabilising ligands. The advantages of using this approach include: (1) the leaf extract acts as both stabilising agent and reducing agent during the synthesis process; (2) the aqueous synthesis process is environmental friendly and produces no toxic waste; and (3) the technique is simple, straight forward and does not require specialised equipment. Furthermore, the antibacterial activity of both the synthesised Au NPs and the leaf extracts were tested against Bacillus subtilis and Escherichia coli using the Kirby-Bauer sensitivity method [36].

\section{Materials and methods}

\section{Chemicals}

The $\mathrm{AuCl}_{4}{ }^{-}$ions were produced by dissolving high purity $\mathrm{Au}$ wire $(99.99 \%)$ in a solution of aqua regia. Both $\mathrm{HNO}_{3}$ and $\mathrm{HCl}$ were purchased from Sigma-Aldrich (Castle Hill, NSW, Australia) and used without further purification. Milli$\mathrm{Q}^{\circledR}$ water was used throughout all synthesis procedures involving aqueous solutions and was produced by a Barnstead Ultrapure Water System D11931 (Thermo Scientific Dubuque IA $18.3 \mathrm{M} \Omega \mathrm{cm}^{-1}$ ).

\section{Leaf material and preparation of leaf extract}

The E. macrocarpa leaves were collected from several locations around the Murdoch University campus in Perth, Western Australia. A wide selection of Mottlecah leaves, ranging
Fig. 1 a Eucalyptus macrocarpa with its distinctive silvery foliage and prominent red flowers, b droplets consisting of: $(i)$ filtered solution containing Au NPs, (ii) solution s1 composed of $\mathrm{AuCl}_{4}{ }^{-}$ and leaf extract solution (1:1), (iii) pure $\mathrm{AuCl}_{4}^{-}$solution and (iv) solution of raw leaf extract
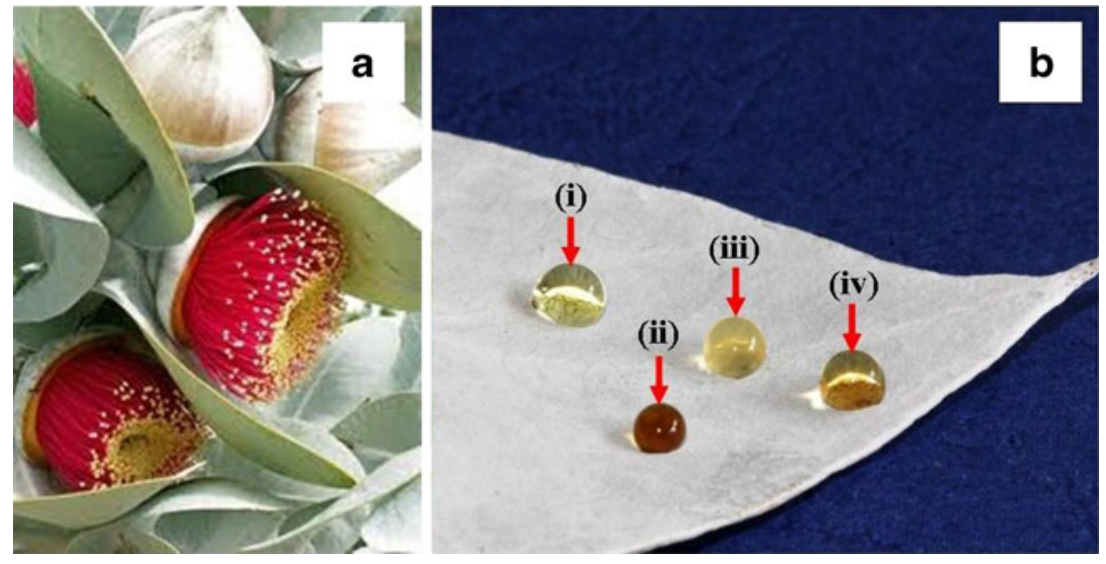
from young to mature leaves, was harvested from various locations on each plant. Generally, five locations were selected (top, north, south, east and west), and on average, ten leaves were taken from each of the locations. Both the adaxial and abaxial sides of the leaf were examined, with only healthy leaves free from damage being harvested. The leaves were washed several times with Milli- ${ }^{\circledR}$ water to remove any dust or debris. After cleaning, $10 \mathrm{~g}$ of Mottlecah leaves were finely cut into small strips and added to a $100 \mathrm{~mL}$ solution of Milli$\mathrm{Q}^{\circledR}$ water. The aqueous mixture was then poured into the blending bowl of an IKA ${ }^{\circledR}$ T25 Digital Ultra Turrax ${ }^{\circledR}$ homogenizer. The mixture was homogenised at 5,000 rpm for $10 \mathrm{~min}$ at a room temperature of $24{ }^{\circ} \mathrm{C}$. At the end of this time, the solution was filtered using a Hirsch funnel to remove leaf debris. This was followed by two further filtrations using a $0.22 \mu \mathrm{m}$ Millex $^{\circledR}$ (33 mm diameter) syringe filter unit. At the end of the three-step filtration procedure, the leaf extract was placed in a glass vial ready for the synthesis of Au NPs.

\section{Removal of epicuticular waxes}

To determine the influence of epicuticular wax in the leaf extract, it was necessary to prepare a leaf extract without wax. This was achieved by first selecting several pre-cleaned leaves (discussed above) and then using a solution of chloroform to wash each leaf. Both the adaxial and abaxial sides of the leaf were washed to remove all surface waxes. The washing procedure consisted of slowly pouring a $50 \mathrm{~mL}$ solution of chloroform over the entire inclined leaf collecting the run off in a small beaker, each side being done in turn. During the $30 \mathrm{~s}$ washing period, the chloroform efficiently removed the epicuticular waxes from the leaf surface. Subsequent scanning electron microscope (SEM) analysis revealed that the waxes had indeed been removed from the leaf surface leaving the underlining cutin undamaged.

\section{Synthesis of gold nanoparticles}

The Au NPs used as the control were synthesised by first adding a $1.0 \mathrm{~mL}$ solution of $1 \mathrm{mM} \mathrm{AuCl}_{4}{ }^{-}$to a $10 \mathrm{~mL}$ solution of Milli-Q ${ }^{\circledR}$ water, while the solution was stirred vigorously. This was followed by adding a $1.0 \mathrm{~mL}$ solution of $1 \mathrm{mM}$ sodium citrate (stabilising and capping agent) to the aqueous solution at room temperature $\left(24^{\circ} \mathrm{C}\right)$. The reduction of the $\mathrm{Au}$ nanoparticles was initiated by the addition of a $1.0 \mathrm{~mL}$ solution of $0.01 \mathrm{M}$ sodium borohydride to the aqueous solution, while the solution was stirred. The reduction process was allowed to proceed at room temperature $\left(24{ }^{\circ} \mathrm{C}\right)$.

The biological reduction of a $1.0 \mathrm{~mL}$ solution of $10 \mathrm{mM}$ aqueous $\mathrm{AuCl}_{4}{ }^{-}$ion solution was investigated using three solutions with varying amounts of leaf extract without wax and with wax. The quantities of leaf extract used to make up the solutions consisted of $1 \mathrm{~mL}$ for $\mathrm{g}_{1}, 2 \mathrm{~mL}$ for $\mathrm{g}_{2}$ and $3 \mathrm{~mL}$ for $\mathrm{g}_{3}$. Once the $\mathrm{AuCl}_{4}{ }^{-}$ion solution was added to each quantity of leaf extract, the solutions were then vigorously stirred for $1 \mathrm{~min}$. The reduction process was allowed to proceed at room temperature $\left(24^{\circ} \mathrm{C}\right)$, with stable $\mathrm{Au}$ NPs being produced within $1 \mathrm{~h}$.

\section{Leaf droplets}

A clean Mottlecah leaf was selected. The leaf was laid flat in the horizontal plane to prevent the various droplet types from rolling off the surface. Then using a fluid-specific clean glass pipette fitted with a rubber bulb, four individual droplets were placed onto the leaf surface. The droplets consisting of: (1) filtered solution containing Au NPs, (2) solution s1 composed of $\mathrm{AuCl}_{4}{ }^{-}$and leaf extract solution, (3) pure $\mathrm{AuCl}_{4}{ }^{-}$solution, and (4) solution of raw leaf extract. The progress of the reduction process was photographed over a 2 $\mathrm{h}$ period using a Canon EDS $600 \mathrm{D}$ digital camera (Canon Inc, Tokyo Japan fitted with macro lens EF $100 \mathrm{~mm} \mathrm{1:2:8}$ USM). A typical photograph taken after $1 \mathrm{~h}$ is presented in Fig. $1 b$.

\section{Characterisation of biologically reduced Au nanoparticles}

All samples were examined and characterised using five advanced analysis techniques. These included: UV-visible spectrum analysis, X-ray diffraction spectroscopy (XRD), energy dispersive X-ray spectroscopy (EDAX), transmission electron microscopy (TEM) and field emission scanning electron microscopy (FESEM).

\section{UV-visible spectrum analysis}

A series of samples were prepared. The first set consisted of three controls: (1) Milli-Q ${ }^{\circledR}$ water, (2) pure $\mathrm{AuCl}_{4}{ }^{-}$solution and (3) pre-filtered pure leaf extract (filtered twice, each time using a new Whatman $0.22 \mu \mathrm{m}$ syringe filter). The test solutions consisted of the three Au colloids $g_{1}, g_{2}$ and $g_{3}$. The UVvisible spectra of each of the samples was then measured using a Varian Cary 50 series UV-visible spectrophotometer version 3 , over a spectral range from 200 to $1,100 \mathrm{~nm}$, with a $1 \mathrm{~nm}$ resolution over the first hour at room temperature of $24^{\circ} \mathrm{C}$.

\section{XRD spectroscopy}

After the end of each reduction procedure, samples for XRD examination were extracted from each glass vial using a clean glass pipette fitted with a rubber bulb. Then two to three drops of each sample were dispersed over the surface of a specific glass microscope slide. Then each glass slide was then dried under vacuum for a period of $4 \mathrm{~h}$. At the end of this time, the dried samples were then characterised using XRD spectroscopy. The XRD spectra were recorded at room 
temperature $\left(22^{\circ} \mathrm{C}\right)$, using a Bruker D8 series diffractometer $\left(\mathrm{Cu} \mathrm{K} \mathrm{K}_{\alpha}=1.5406 \AA\right.$ radiation source $)$ operating at $40 \mathrm{kV}$ and $30 \mathrm{~mA}$. The diffraction patterns were collected over a $2 \theta$ range from 15 to $80^{\circ}$, with an incremental step size of $0.04^{\circ}$ using flat plane geometry. The acquisition time was $2 \mathrm{~s}$. The powder XRD spectrum was used to identify the size of the $\mathrm{Au}$ particles and their crystalline structure. The particle size was calculated using the Debye-Scherrer equation (Eq. 2) from the respective XRD patterns and estimated from both TEM and FESEM images.

\section{EDAX spectroscopy}

Each sample for EDAX examination was initially deposited onto a thin mica strip using a glass pipette, the mica strip was attached to a SEM stub using carbon tape. The samples were then dried under vacuum overnight. The following day, all samples were sputter-coated with a 3-nm layer of platinum. The samples were then examined using an Oxford Instruments EDS X-ray detector (EDAX) and Oxford Instruments energy dispersive X-ray detectors (EDS). The electron backscatter diffraction was used during the analysis, and the EDS aperture was set to $60 \mu \mathrm{m}$ and operated at $20 \mathrm{kV}$.

\section{TEM}

The size and morphology of the Au NPs was investigated using TEM. Sample preparation consisted of filtering the suspensions two times, each time using a new Whatman $0.22 \mu \mathrm{m}$ syringe filter. After filtration, a single drop from each sample was deposited onto its respective carbon-coated copper TEM grid using a micropipette and then allowed to slowly dry over a 24 -h period. After sample preparation, a bright field TEM study was carried out using a Phillips CM100 electron microscope (Phillips Corporation Eindhoven, The Netherlands) operating at $80 \mathrm{kV}$.

\section{FESEM}

Each sample for FESEM examination was initially deposited onto a thin mica strip using a glass pipette; the mica strip was attached to a SEM stub using carbon tape. The samples were then dried under vacuum overnight. The following day, all samples were sputter-coated with a 3-nm layer of platinum. The particle size and morphological features of the samples were investigated using a high resolution FESEM (Zeiss Neon 40EsB FIBSEM) at $5 \mathrm{kV}$, with a $30-\mu \mathrm{m}$ aperture operating under a pressure of $1 \times 10^{-10}$ Torr.

Microbial sensitivity of Au nanoparticles

The antibacterial activity of the biologically reduced Au NPs was investigated using the sensitivity method of Kirby-
Bauer [36]. In this technique, the test media was composed of Difco nutrient agar $(23 \mathrm{~g} / 1,000 \mathrm{~mL})$, agar $(5 \mathrm{~g} / 1,000 \mathrm{~mL})$, yeast extract $(4 \mathrm{~g} / 1,000 \mathrm{~mL})$ and distilled water $(1,000 \mathrm{~mL})$. The components were thoroughly mixed via heating and agitation to completely dissolve the components. The medium was then autoclaved at $121^{\circ} \mathrm{C}$ for $15 \mathrm{~min}$. At the end of the autoclaving period, a $20-\mathrm{mL}$ volume of the media was added to each Petri dish ( $90 \mathrm{~mm}$ diameter) and then allowed to solidify at room temperature before being stored at $4{ }^{\circ} \mathrm{C}$ for future use.

Two bacteria, namely E. coli and B. subtilis were used to investigate the antibacterial activity of the Au NPs. In each case, the respective bacteria were cultured in a media composed of Difco nutrient broth $(8 \mathrm{~g} / 1,000 \mathrm{~mL})$, yeast extract $(4 \mathrm{~g} / 1,000 \mathrm{~mL})$ and distilled water $(1,000 \mathrm{~mL})$. The nutrient medium was then autoclaved at $121{ }^{\circ} \mathrm{C}$ for $15 \mathrm{~min}$. After autoclaving, the nutrient medium was ready for culturing bacteria; in the first stage, each respective bacteria was added to a $10-\mathrm{mL}$ sample of the nutrient medium and then incubated overnight at $28{ }^{\circ} \mathrm{C}$ while being agitated. In the second stage, the cultures were then added to a $100-\mathrm{mL}$ solution of nutrient media and further incubated overnight at $28{ }^{\circ} \mathrm{C}$, while being agitated. The following day, a $1-\mathrm{mL}$ sample of the culture medium was pipette onto the surface of the nutrient containing Petri dish. The flooded dish is then carefully tilted in various orientations, until the entire nutrient surface was covered. Once the nutrient surface was covered, the excess culture medium was carefully removed using a pipette. Then, the cover was placed on each Petri dish, inverted and left to dry for $45 \mathrm{~min}$.

While the Petri dishes were drying, $25 \mu \mathrm{g} / \mathrm{mL}$ solutions of as prepared Au NPs (Au NPs control and leaf extract solution (with and without wax) and leaf extract + Au NPs solution $\mathrm{g}_{1}$ (with and without wax)) were transferred onto a sterile 6-mm diameter Whatman AA (2017-006) sample disc using a pipette, and then allowed to dry for $20 \mathrm{~min}$. After drying, sterile forceps were used to place the sample disc onto the centre of the inoculated Petri dish. After all the samples were placed in their respective inoculated dishes, the covers were fitted and then the dishes were incubated overnight at $28{ }^{\circ} \mathrm{C}$. After incubation, the diameter of the bacterial inhibition zone of each sample was measured using a standard scale ruler with an accuracy of $\pm 0.5 \mathrm{~mm}$, with all measurements being rounded up to the next whole millimetre. All sample experiments were carried out in triplicate and the mean inhibition zone diameters recorded.

In addition, optical microscopy was used throughout the studies to examine the bacterial inhibition zone. An Olympus BX51 compound microscope (Olympus Optical Co. Ltd., Tokyo, Japan) was used for all optical studies, and photographs were taken using the DP 70 camera attachment. 


\section{Results and discussions}

This preliminary study reports the green synthesis of Au NPs by the biological reduction of aqueous $\mathrm{AuCl}_{4}{ }^{-}$using the leaf extract from E. macrocarpa (Mottlecah). The water soluble ingredients present in the leaf extract were found to be both a highly effective reducing agent and an efficient stabilising agent. The untreated leaf extract was found to be acidic, with a $\mathrm{pH}$ of 5 for leaf extract without wax and a $\mathrm{pH}$ of 5.5 with wax. The formation of the Au NPs could be easily monitored by the change in colour of the reactive mixture. The synthesis of Au NPs begins with the addition of the light yellow $\mathrm{AuCl}_{4}{ }^{-}$aqueous solution $(\mathrm{pH}=1)$ to a solution of raw leaf extract forming the reactive mixture, which typically had a $\mathrm{pH}$ of 2. At this point, the reactive mixture is subjected to $10 \mathrm{~min}$ of homogenization. During homogenization, the prevailing process is Au metal nucleation, and the mixture changes from a light yellow colour to a pale brown colour due to the excitation of surface plasmon vibrations of the forming Au NPs. After the initial homogenization treatment, the reaction mixture was then allowed to stand, and over the next 60 min further reduction of $\mathrm{Au}^{\mathrm{III}}$ took place. During this period, the colour of the complex slowly darkens to a deeper brown colour, indicating the increasing numbers of $\mathrm{Au}$ nuclei steadily forming. The formation of Au NPs was confirmed by both UV-visible spectrum analysis and EDAX spectroscopy analysis for all three solutions $\left(\mathrm{g}_{1}, \mathrm{~g}_{2}\right.$ and $\left.\mathrm{g}_{3}\right)$. UV-visible spectrum analysis for the solutions indicated that the maximum absorbance occurred at $570 \mathrm{~nm}$, which is similar to the maximum absorbance of $573 \mathrm{~nm}$ reported by Philip using the leaf extract from Hibiscus rosa sinensis and the maximum absorbance of $560 \mathrm{~nm}$ reported by Singh et al. using the leaf extract of ginger (Zingiber officinale) [9, 37]. A representative UV-visible spectrum analysis for solution $\mathrm{g}_{1}(1: 1)$ is presented in Fig. 2a, while the corresponding EDAX spectroscopy analysis presented in Fig. 2b clearly indicates the presence of metallic $\mathrm{Au}$. The remaining two solutions $\left(g_{2}\right.$ and $\left.g_{3}\right)$ gave similar UV-visible and EDAX results. The net result of these two analysis techniques confirmed the formation of Au NPs and can be summarised in Eq. 1.

$$
\mathrm{AuCl}_{4}{ }^{-}+\text {Mottlecah leaf extract } \rightarrow \text { Stabilised Au NPs }
$$

TEM images taken after $60 \mathrm{~min}$ of biological reduction reveal that products present in the aggregate are NPs, with the main product being spherical particles ranging in size from 20 to $80 \mathrm{~nm}$. However, coexisting with the spheres are $\mathrm{Au}$ nanometre-sized crystalline shapes (equilateral or truncated triangular, pentagon and hexagonal), all ranging in size from 50 to $100 \mathrm{~nm}$, see Fig. 3a and b for representative images of the particle aggregates. This is unlike the result for Au NPs synthesised using sodium borohydride, which only produced spherical particles with a mean diameter of $30 \mathrm{~nm}$. During biological reduction, NPs are produced during the first $60 \mathrm{~min}$, after this initial period, nanometre scale crystalline shapes steadily grow into micrometre scale plates and spheres. After $120 \mathrm{~min}$, micrometre-sized particles are the dominant particles found in the aggregate. Figure 4(a) presents a representative aggregate of micrometre-sized particles and plates. Triangular and hexagonal crystalline shapes are interspersed within the aggregate and are characterised by their smooth plate-like structure, which was also seen in the TEM images presented in Fig. 3a and b. Also presented in Fig. 4 are two colourised FESEM images (b) and (c), which highlight the morphology of the hexagonal and truncated triangular Au plates. The side lengths of the truncated triangular-shaped plates can generally reach a length of $6 \mu \mathrm{m}$, while the side lengths of the hexagonal-shaped plates are typically around $4 \mu \mathrm{m}$. The thickness of both plate types was found to vary from 300 to $600 \mathrm{~nm}$. The aggregate was also found to have spherical particles with diameters ranging size from $200 \mathrm{~nm}$ up to $1.5 \mu \mathrm{m}$.

To investigate the crystalline nature of the Au NPs and nanometre scale platelets, dried Au powder samples were investigated using XRD spectroscopy. The Au crystalline phases present were found to be consistent with phases incorporated in the International Centre for Diffraction Data databases. A typical XRD pattern of a dried Au powder sample is presented in Fig. 3c. Inspection of Fig. 3c reveals the presence of intense peaks located at 38.3, 44.5, 64.5 and $77.9^{\circ}$, which correspond to the main (hkl) indices for $\mathrm{Au}$ : (111), (200), (220) and (311). The Bragg peaks indicate that the synthesised Au NP based powder was composed of pure crystalline metallic Au consisting of an fcc lattice structure. The intense peak at (111) indicates that this plane was the predominant orientation, and this was confirmed by evaluating the peak intensity ratio of the (200) and (111) peaks, which gave a value of 0.18 . The value of 0.18 is much lower than the conventional bulk intensity ratio of 0.52 normally associated with $\mathrm{Au}$, and clearly indicates that the (111) plane is indeed the predominant orientation found in the synthesised Au powders. The dominance of the (111) plane resulting from the biosynthesis of Au NPs has also been reported by Philip using $H$. rosa sinensis [9] and Zhang et al. using chloroplasts [38]. The crystalline size, $\mathrm{t}_{(\mathrm{hkl})}$, of $\mathrm{Au}$ NPs was calculated from the XRD pattern using the Debye-Scherrer equation presented in Eq. 2 below:

$t_{(h k l)}=\frac{0.9 \lambda}{B \cos \theta_{(h k l)}}$

where, $\lambda$ is the wavelength of the monochromatic X-ray beam, $B$ is the full width at half maximum (FWHM) of the peak at the maximum intensity, $\theta_{(\mathrm{hkl})}$ is the peak diffraction angle that satisfies Bragg's law for the (h k l) plane, and $t_{(\mathrm{hkl})}$ 
Fig. 2 a A representative UVvisible spectrophotometer analysis of Au NPs $\left(g_{1}\right)$ synthesised using fresh Eucalyptus macrocarpa leaves, b corresponding EDAX spectra of sample
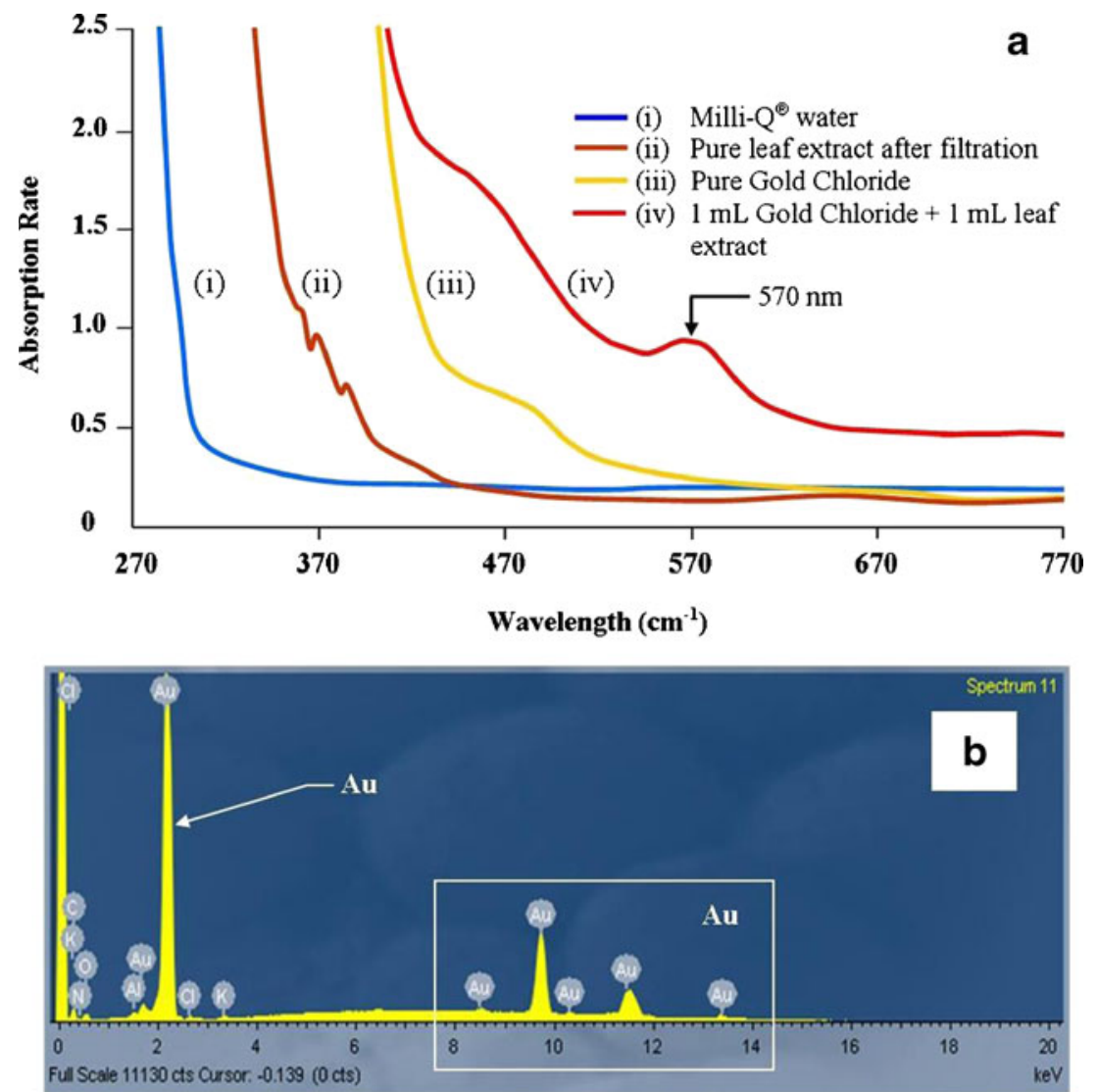

is the crystallite size. An estimate of the mean crystallite size using the FWHM of the (111) peak was calculated to be $84 \mathrm{~nm}$. The TEM analysis revealed a particle size range from 20 to
$100 \mathrm{~nm}$, with the aggregation containing not only sphericalshaped particles but also truncated triangular, pentagon and hexagonal-shaped crystalline particles, see Fig. $3 a$ and $b$.

Fig. 3 a and $\mathbf{b}$ Representative TEM micrographs of Au NPs synthesised using leaf extracts with wax $(L W)$ from Eucalyptus macrocarpa leaves, $\mathbf{b}$ a typical XRD pattern of Au NPs synthesised from leaf extracts
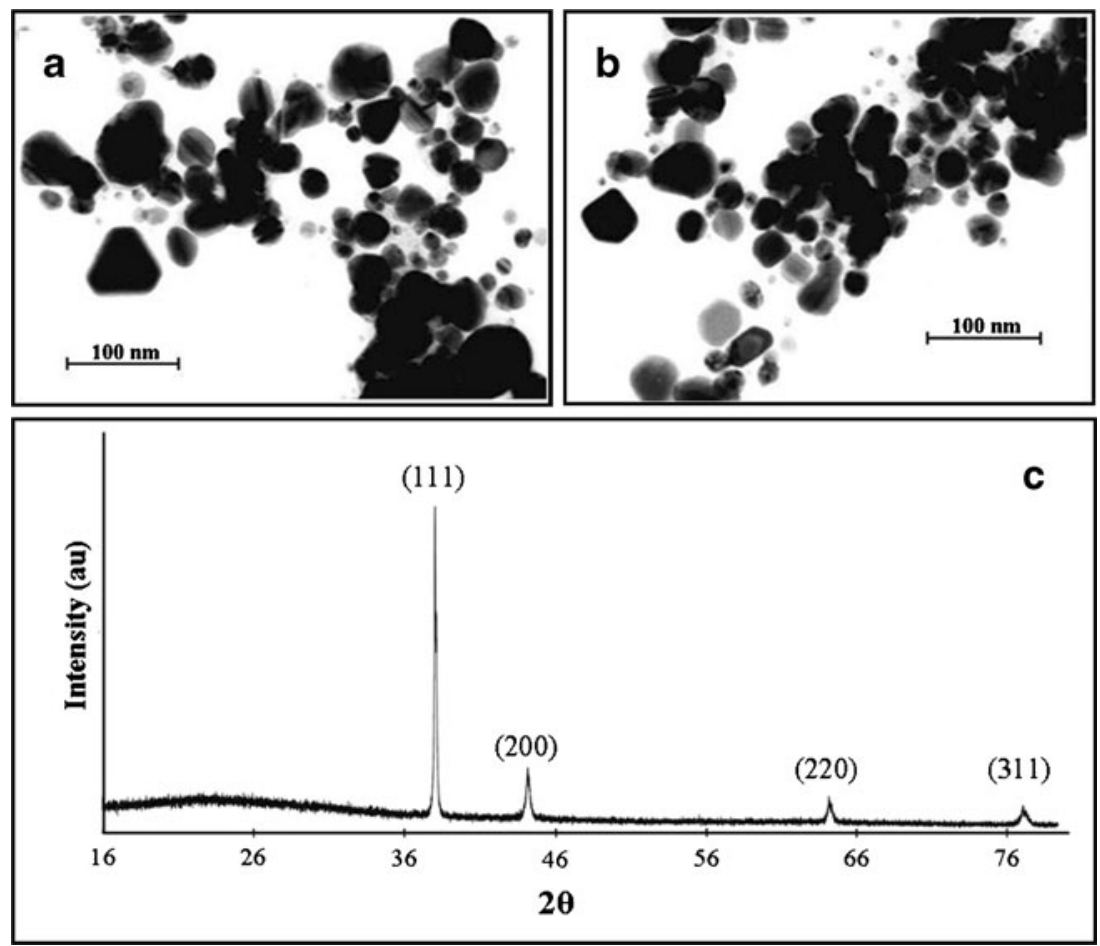
Fig. 4 a A representative FESEM image of an aggregate containing Au particles and plates synthesised in leaf extract with wax $(L W)$ over periods greater than $1 \mathrm{~h}$; $\mathbf{b}$ and $\mathbf{c}$ colourised FESEM images highlighting truncated triangular and hexagonal $\mathrm{Au}$ plates
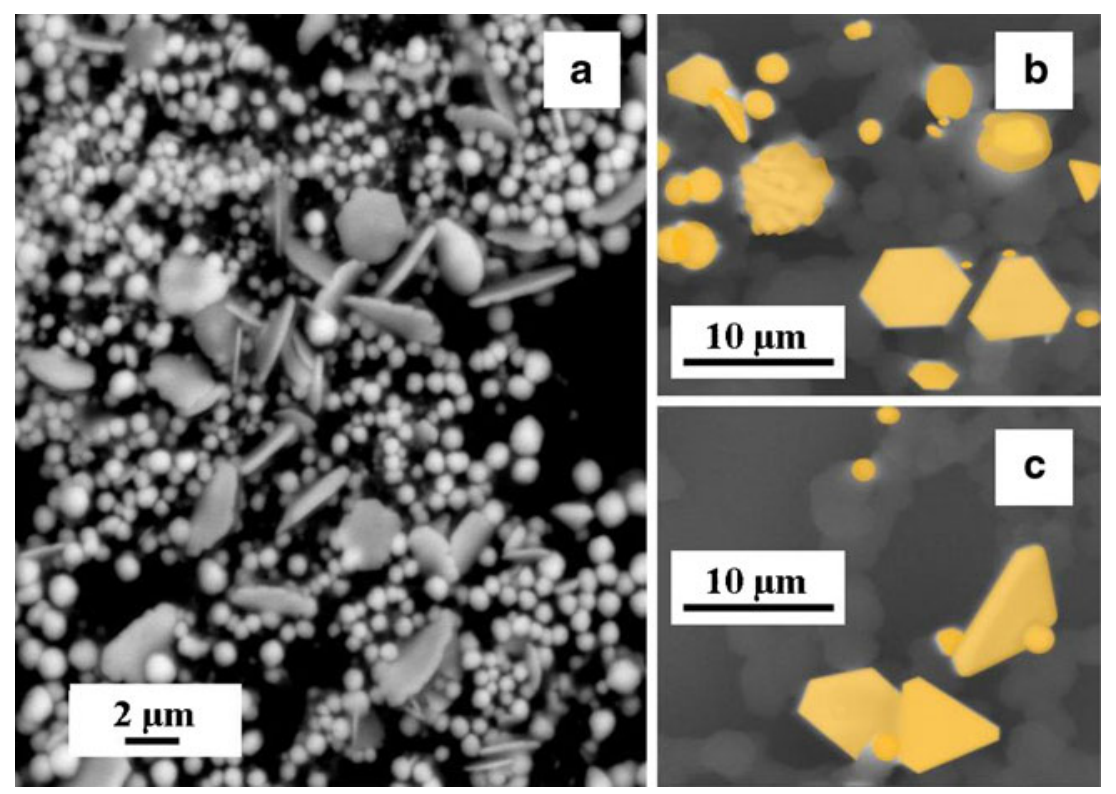

A possible mechanism to explain the plate-like growth and morphology of particles during reduction in the leaf extract could be the result of competitive growth between crystallographic surfaces, which results in a preferential attachment in a particular orientation [39]. An alternative mechanism proposed by Wang et al. arises from the initial spontaneous self-assembly of particles along a particular crystallographic orientation. This is followed by other $\mathrm{Au}$ particles coalescing at the planar interface, which effectively reduces their surface energy and contributes to the growth of the particle along this particular orientation [40]. In this study, analysis of the XRD data clearly indicates the dominance of the (111) orientation and confirms preferential particle growth along (111) crystal plane. This explains the inherent anisotropy of the atomically flat surface of the $\mathrm{Au}$ crystalline-shaped plates produced initially in the nanometre range. Subsequent growth in the micrometre range also produces the atomically flat surfaces, which are characteristic of the plate-like particles synthesised using E. macrocarpa leaf extracts.
Fig. 5 a Zone of inhibition produced by colloid $\mathrm{g}_{1}[\mathrm{Au}$ NPs+leaf extract (with wax)] for Escherichia coli diameter $29 \mathrm{~mm}$, b microscopic view of the zone of inhibition boundary, c antibacterial activity of $\mathrm{Au}$ NPs against Bacillus subtilis and $\mathbf{d}$ antibacterial activity of Au NPs against Escherichia coli
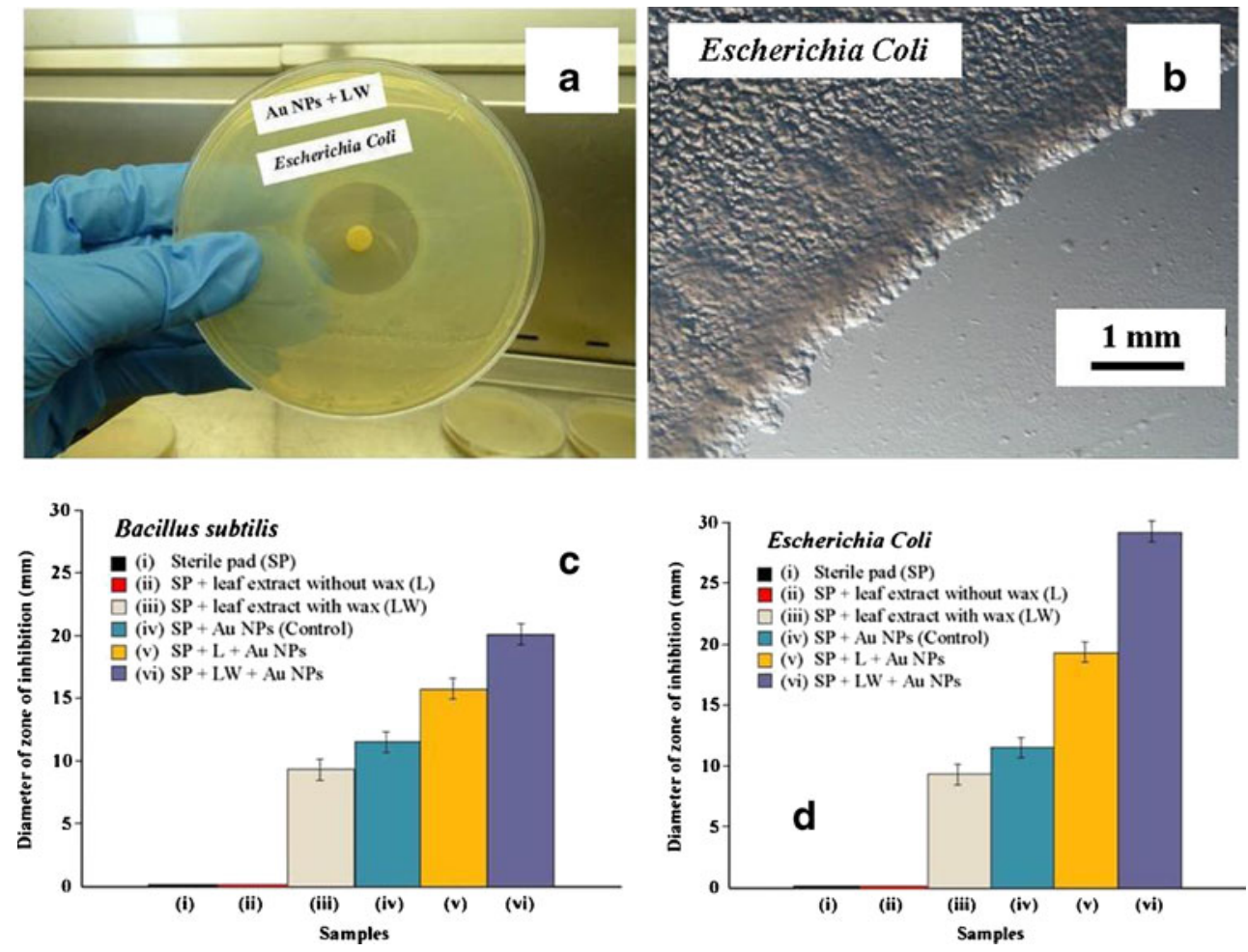
The antibacterial potential of the Au NPs synthesised using E. macrocarpa leaf extracts, the leaf extract itself and a combination of both (synergistic effects) were tested against $B$. subtilis (gram-positive) and E. coli (gram-negative). The leaf extract was initially tested against the two bacteria because earlier studies by Murata et al. were able to show an antibacterial effect of a compound (macrocarpal A) isolated from the leaf extract [41]. Further studies on the E. macrocarpa leaves by Yamakoshi et al. revealed the presence of a further six antibacterial compounds (macrocarpal B to G) [42]. Their study also determined the structure of the macrocarpal compounds from both XRD and NMR analysis. The analysis revealed that the compounds were composed of phloroglucinol dialdehyde diterpene derivatives. At this stage, it is not known if any of these compounds are directly involved in the biological reduction of the Au NPs, but there is clear evidence that these compounds are giving the leaf extract its antibacterial properties. For example, Petri dishes with only a sterile pad (control) and dishes with a sterile pad treated with leaf extract (without wax) recorded no zone of inhibition. However, dishes with a sterile pad treated with leaf extract (with wax) recorded a zone of inhibition of $9 \mathrm{~mm}$ for both $B$. subtilis and E. coli. The results are graphically presented in Fig. 5c (B. subtilis) and d (E. coli).

In the case of the Au NPs, the first tested was the Au NP (control), which was synthesised using sodium borohydride as the reducing agent and sodium citrate as the stabilising and capping agent. The Petri dishes containing a sterile pad treated with Au NPs (control) recorded a zone of inhibition of $12 \mathrm{~mm}$ for both B. subtilis and E. coli. The results of the zone of inhibition measurements indicate that both bacteria are resistant to the Au NP control. In the next step, Au NPs synthesised using the leaf extract were tested against the two bacteria. The Petri dishes containing a sterile pad treated with Au NPs+leaf extract (without wax) recorded a zone of inhibition of $16 \mathrm{~mm}$ for B. subtilis and $19 \mathrm{~mm}$ for E. coli. Since the leaf extract without wax was found to have no zone of inhibition (no antimicrobial effect), the larger zones of inhibition recorded for both bacteria are the result of the bio-reduced Au particles. This result indicates that both bacteria are more sensitive to the bio-reduced Au particle. And in the final test set, Petri dishes containing a sterile pad treated with Au NPs+leaf extract (with wax) recorded a zone of inhibition of $20 \mathrm{~mm}$ for $B$. subtilis and $29 \mathrm{~mm}$ for E. coli. The results of the Au NPs (control) and Au NPs synthesised using leaf extract are graphically presented in Fig. 5c (B. subtilis) and d (E. coli).

Inspection of both Fig. $5 \mathrm{c}$ and d reveals that the size of the diameters obtained for the zone of inhibition are much larger for $\mathrm{Au} \mathrm{NPs+leaf} \mathrm{extract} \mathrm{(with} \mathrm{wax)} \mathrm{than} \mathrm{those} \mathrm{without} \mathrm{leaf}$ extract. It is also evident that there is a synergistic effect between the Au NPs and the leaf extract. This is dramatically seen for Au NPs+leaf extract (with wax) against $E$. coli (zone of inhibition is $29 \mathrm{~mm}$ ), which was significantly greater than the $19 \mathrm{~mm}$ for $\mathrm{Au} \mathrm{NPs}+$ leaf extract (without wax). The results also show that Au NPs+leaf extract (with wax) was much more effective against the gram-negative $E$. coli (diameter $29 \mathrm{~mm}$ ) than the gram-positive $B$. subtilis (diameter $20 \mathrm{~mm}$ ). A photograph of the $29-\mathrm{mm}$ zone of inhibition for E. coli resulting from $\mathrm{Au}$ NPs+leaf extract (with wax) is presented in Fig. 5a, while an enlarged microscopic view of the interface between $E$. coli and the zone of inhibition is presented in Fig. 5b. The results of the antibacterial study reveal that both $B$. subtilis (gram-positive) and $E$. coli (gram-negative) were sensitive to Au NPs+leaf extract (with wax). The leaf extract without wax proved to have no antibacterial effect. However, leaf extract with wax produced a small zone of inhibition $(9 \mathrm{~mm})$ for both bacteria, indicating they were both resistant to the leaf extract. The results indicate that the antibacterial properties contained within the leaf are resident in the waxes, and when the waxes are combined with Au NPs, a significant synergistic effect was produced. Combining the inherent properties of the leaf wax with those of Au NPs has proven to be beneficial in producing an effective antibacterial agent against both B. subtilis and E. coli.

\section{Conclusion}

A straightforward, clean and environmentally friendly method of biologically synthesising Au NPs was demonstrated using the leaf extracts from an indigenous Australian plant $E$. macrocarpa. The leaf extracts acted as both the stabilising agent and the reducing agent, with the process occurring at room temperature. The biological reduction of $\mathrm{AuCl}_{4}{ }^{-}$produced an aggregate of NPs consisting of spherical particles ranging in size from 20 to $80 \mathrm{~nm}$ and nanometre-sized crystalline shapes (equilateral or truncated triangular, pentagon and hexagonal), ranging in size from 50 to $100 \mathrm{~nm}$. Longer reduction times allowed the NPs to grow into micrometresized particles of varying size and morphology. The sensitivity method of Kirby-Bauer was used to establish a synergistic effect between the Au NPs and the waxes contained in the leaf extracts. And when combined, the Au NPs and leaf extracts (with wax) created an effective antibacterial agent against both B. subtilis and E. coli.

Acknowledgments This work was partly supported by the Western Australian Nanochemistry Research Institute (WANRI). The authors would also like to thank Mr Ravi Krishna Brundavanam for his assistance with the XRD measurements and Mrs. Monaliben Shah, Mrs. Sridevi Brundavanam and Dr Michelle Buttery for their assistance in the antibacterial studies.

Conflict of interest The authors claim no conflict of interest in this work.

Open Access This article is distributed under the terms of the Creative Commons Attribution License which permits any use, distribution, and reproduction in any medium, provided the original author(s) and the source are credited. 


\section{References}

1. Sperling RA, Gil PR, Zhang F, Zanella M, Parak WJ (2008) Biological applications of gold nanoparticles. Chem Soc Rev 37:1896-1908

2. Sykora D, Kasicka V, Miksik I, Rezanka P, Zaruba K, Matejka P, Kral V (2010) Applications of gold nanoparticles in separation sciences. J Sep Sci 30:372-387

3. Cai W, Gao T, Hong H, Sun J (2008) Applications of gold nanoparticles in cancer nanotechnology. Nanotechnol Sci Appl 1: $17-32$

4. Pankhurst QA, Connolly J, Jones SK, Dobson J (2003) Applications of magnetic nanoparticles in biomedicine. J Phys D: Appl Phys 36:R167-R181

5. Yan H, Park SH, Finkelstein G, Reif JH, LaBean TH (2003) DNAtemplated self-assembly of protein arrays and highly conductive nanowires. Science 301:1882-1884

6. Keren K, Berman RS, Buchstab E, Sivan U, Braun E (2003) DNAtemplated carbon nanotube field-effect transistor. Science 302: $1380-1382$

7. Ghosh SK, Pal T (2007) Interparticle coupling effect on the surface plasmon resonance of gold nanoparticles: from theory to application. Chem Rev 107:4797-4862

8. Ai J, Biazar E, Jafarpour M et al (2011) Nanotoxicology and nanoparticle safety in biomedical designs. Int $\mathrm{J}$ Nanomedicine 6:1117-1127

9. Philip D (2010) Green synthesis of gold and silver nanoparticles using Hibiscus rosa sinensis. Physica E 42:1417-1424

10. Kumar P, Singh P, Kumari K et al (2011) A green approach for the synthesis of gold nanotriangles using aqueous leaf extract of Callistemon viminalis. Mater Lett 65:595-597

11. Lengke M, Southam G (2006) Bioaccumulation of gold by sulphate-reducing bacteria cultured in the presence of gold (I)thiosulfate complex. Acta 70(14):3646-3661

12. Joerger TK, Joerger R, Olsson E, Granqvist CG (2001) Bacteria as workers in the living factor: metal-accumulating bacteria and their potential for materials science. Trends Biotechnol 19(1):15-20

13. Ahmad A, Senapati S, Khan MI et al (2003) Intracellular synthesis of gold nanoparticles by a novel alkalotolerant actinomycete Rhodococcus species. Nanotechnology 14:824-828

14. Kuber C, Souza SF (2006) Extracellular biosynthesis of silver nanoparticles using the fungus Aspergillus fumigates. Colloids Surf B 47:160-164

15. Ahmad A, Senapati S, Khan MI et al (2003) Extracellular biosynthesis of monodisperse gold nanoparticles by a novel extremophilic actinomycete Thermomonospora species. Langmuir 19:3550-3553

16. Simkist K, Wilbur KM (1989) Mulluscs-epithelial control of matrix and minerals. In: Simkist K, Wilbur KM (eds) Biomineralisation, cell biology and mineral deposition. Academic, New York, pp 231-256

17. Kreibig U, Vollmer M (1995) Optical Properties of Metal Clusters. Springer, Berlin

18. Liu X, Dai Q, Austin L, Coutts J et al (2008) A one-step homogeneous immunoassay for cancer biomarker detection using gold nanoparticle probes coupled with dynamic light scattering. J Am Chem Soc 130:2780-2782

19. Chan WCW, Nie SM (1998) Quantum dot bioconjugates for ultrasensitive nonisotopic detection. Science 281:2016-2018

20. Huang X, Jian PK, El-Sayed IH et al (2006) Determination of the minimum temperature required for selective photothermal destruction of cancer cells with the use of immune-targeted gold nanoparticles. Photochem Photobiol 82:412-417

21. Paciotti GF, Mayer L, Weinreich D et al (2004) Colloidal gold: a novel nanoparticle vector for tumour directed drug delivery. Drug Deliv 11:169-183
22. Sondi I, Salopek-Sondi B (2004) Silver nanoparticles as antimicrobial agent: a case study on $E$. coli as a model for gram-negative bacteria. J Colloid Interface Sci 275:177-182

23. Hsiao M, Chen S, Shieh D, Yeh C (2006) One-pot synthesis of hollow $\mathrm{Au} 3 \mathrm{Cu} 1$ spherical-like and biomineral botallackite $\mathrm{Cu} 2$ $(\mathrm{OH}) 3 \mathrm{Cl}$ flowerlike architectures exhibiting antimicrobial activity. J Phys Chem B 110:205-210

24. Priester J, Stoimenov P, Mielke R, Webb S et al (2009) Effects of soluble cadmium salts versus CdSe quantum dots on the growth of planktonic Pseudomonas aeruginosa. Environ Sci Technol 43:2589-2594

25. Brayner R, Ferrari-Iliou R, Brivois N, Djediat S, Benedetti M, Fiévet $\mathrm{F}$ (2006) Toxicological impact studies based on Escherichia coli bacteria in ultrafine $\mathrm{ZnO}$ nanoparticles colloidal medium. Nano Lett 6:866-870

26. Simon-Deckers A, Loo S, Mayne-L'hermite M, Herlin-Boime N, Menguy N, Reynaud C, Gouget B, Carrie M (2009) Size-, composition- and shape-dependent toxicological impact of metal oxide nanoparticles and carbon nanotubes toward bacteria. Environ Sci Technol 43:8423-8429

27. Niemeyer CM (2001) Nanoparticles, proteins and nucleic acids: biotechnology meets materials science. Angew Chem Int Ed 40:4128-4158

28. Hirsch LR et al (2003) Nanoshell-mediated near-infrared thermal therapy of tumours under magnetic resonance guidance. Proc Natl Acad Sci U S A 100:13549-13554

29. Zheng Y, Sache L (2009) Gold nanoparticles enhance DNA damage induced by anti-cancer drugs and radiation. Radiat Res 172:114-119

30. Cheng Y, Samia AC, Li J et al (2010) Delivery and efficacy of a cancer drug as a function of the bond to the gold nanoparticle surface. Langmuir 26:2248-2255

31. Thomas M, Klibanov AM (2003) Conjugation to gold nanoparticles enhances polyethylenimine's transfer of plasmid DNA into mammalian cells. Proc Natl Acad Sci U S A 100:9138-9143

32. Mukherjee P, Bhattacharya R, Bone N et al (2007) Potential therapeutic application of gold nanoparticles in B-chronic lymphocytic leukaemia (BCLL): enhancing apoptosis. J Nanobiotechnol. doi:10.1186/1477-3155-5-4

33. Hernandez-Sierra JF, Ruiz F, Pena DC et al (2008) Antimicrobial sensitivity of Streptococcus mutans to nanoparticles of silver, zinc oxide and gold. Nanomedicine NBM 4:237-240

34. http://eol/pages/635316/overview Encyclopaedia of Life: Eucalyptus Macrocarpa. Last accessed January 212013.

35. Poinern GEJ, Le XT, Fawcett D (2011) Super-hydrophobic nature of nanostructures on an indigenous Australian eucalyptus plant and its potential application. Nanotechnol Sci Appl 4:113-121

36. Jorgensen JH, Turnidge JD (2007) Susceptibility test methods: dilution and disc diffusion methods. In: Murray PR, Baron EJ et al (eds) Manual of clinical microbiology, 9th edn. ASM Press, Washington DC, pp 1152-1172

37. Singh C, Sharma V, Naik PK et al (2011) A green biogenic approach for synthesis of gold and silver nanoparticles using Zingiber officinale. Dig J Nanomater Biostruct 6(2):535-542

38. Zhang YX, Zheng J, Gao G et al (2011) Biosynthesis of gold nanoparticles using chloroplasts. Int J Nanomedicine 6:2899-2906

39. Alexandrides P (2011) Gold nanoparticle synthesis, morphology control and stabilisation facilitated by functional polymers. Chem Eng Technol 34(1):15-28

40. Wang L, Chen X, Zhan J et al (2005) Synthesis of gold nano and microplates in hexagonal liquid crystals. J Phys Chem B 109:3189-3194

41. Murata M, Yamakoshi et al (1990) Macrocarpal A, a novel antibacterial compound from Eucalyptus macrocarpa. Agric Biol Chem 54(12):3221-3226

42. Yamakoshi Y, Murata M, Shimizu A et al (1992) Isolation and characterization of macrocarpals B-G Antibacterial compounds from Eucalyptus macrocarpa. Biosci Biotechnol Biochem 56(10): $1570-1576$ 